\title{
Adimensionalización de una ecuación diferencial en un problema práctico de ingeniería
}

\author{
Rogelio Alvarado*, Miguel Angel Bernal*^, José Rafael Narváez ${ }^{\star \star \star}$, César \\ Augusto Quiñones ${ }^{\dagger}$ \\ Institución Universitaria Politécnico Grancolombiano
}

FECHA DE ENTREGA: 6 DE NOVIEMBRE DE 2013

FECHA DE EVALUACIÓN: 9 DE MARZO DE 2014

FECHA DE APROBACIÓN: 10 DE MAYO DE 2014

\begin{abstract}
Resumen En este trabajo se muestra la aplicación del teorema Pi de Buckingham para obtener parámetros adimensionales en el proceso de adimensionalizar una ecuación y una consecuencia de esto para el caso particular de un amortiguador elástico conectado al parachoques de un automóvil en la búsqueda de valores óptimos para las constantes de elasticidad del resorte $\kappa$ y de atenuación del amortiguador $c$. Problema estudiado por el ingeniero D. A. Peters en su artículo Optimum Spring-Damper Design for Mass Impact[1]. Por claridad con el lector nos permitimos expandir algunos de los cálculos propuestos en la mencionada publicación y el uso del lenguaje de programación $\mathrm{C}++$ para implementar el método de Newton-Raphson. Finalmente se muestra el instrumento virtual Labview 8.5 que se construyó para el sistema con el objetivo de obtener los mejores valores de $\kappa$ y $c$.
\end{abstract}

\begin{abstract}
This work shows an application of Buckingham Pi theorem to obtain dimensionless parameters from a differential equation used to model an elastic damper system attached to the bumper of a car. Although the original problem was studied by D. A. Peters in his article Optimum Spring-Damper Design for Mass Impact [1], we present to the reader expanded calculations, and computational tools such as $\mathrm{C}++$ and Labview 8.5 are used to obtain optimal values for the spring constant $\kappa$, and the damping constant $c$ of the system as a function of the impact speed, mass vehicle and expected displacement of the bumper.
\end{abstract}

Palabras Clave: Teorema Pi de Buckingham, parámetros adimensionales, instrumento virtual.

Keywords: Buckingham Pi theorem, dimensionless parameters, virtual instrument.

\footnotetext{
* ralvarad@poligran.edu.co

** mabernal@poligran.edu.co

$\star \star \star *$ jnarvaez@poligran.edu.co

$\dagger$ caquinones@poligran.edu.co
} 


\section{Introducción}

Como agente fundamental en el desarrollo de una sociedad el automóvil es uno de los mayores aportes de la ingeniería. Su papel es vital desde el punto de vista económico y cultural debido a la siempre creciente necesidad de un transporte rápido, seguro, confortable, económico y masivo.

El avance tecnológico, el descubrimiento de nuevos materiales y las exigencias de altos estándares de calidad en seguridad, confort y desempeño impactan directamente muchos aspectos de la industria automotriz, lo que hace primordial el continuo estudio e investigación para lograr mejoras en estos aspectos. Pero la seguridad, confort y eficiencia dependen en gran medida de los sistemas de amortiguamiento. El profesor D. A. Peters en su artículo Optimum Spring-Damper Design for Mass Impact[1] optimiza los parámetros del resorte y amortiguador de un automóvil, resolviendo la ecuación diferencial asociada a este sistema para el caso de pequeños impactos.

\section{Descripción del modelo amortiguador-elástico}

El principal objetivo del modelo amortiguador-elástico en el parachoques de un automóvil, figura 1, radica en que al momento del choque frontal la fuerza máxima del impacto sobre el vehículo se atenúe lo máximo posible para un valor mínimo del desplazamiento del parachoques, es decir lograr que una porción significativa de la energía liberada en el choque sea disipada en el amortiguador-elástico, lo que permite que el vehículo sufra menos daños en su estructura protegiendo la integridad de los ocupantes.

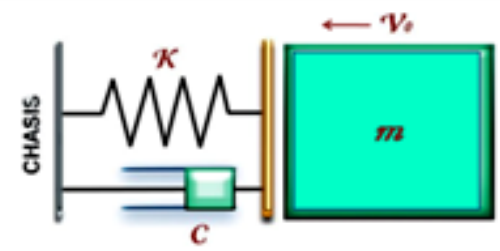

Figura 1. Modelo físico del amortiguador elástico.

El modelo matemático que se obtiene al aplicar las leyes de la mecánica al problema físico planteado es una ecuación diferencial de segundo orden con coeficientes indeterminados $\kappa$ y $c$, relacionados con la elasticidad del resorte y el coeficiente de fricción del amortiguador respectivamente.

$$
\frac{d^{2} x}{d t^{2}}+\frac{c}{m} \frac{d x}{d t}+\frac{\kappa}{m} x=0 .
$$

Las condiciones iniciales del problema son: 
- En el momento del impacto $(t=0)$, el parachoques se encuentra en su posición de equilibrio $x=0$.

- En el momento del impacto $(t=0)$, la velocidad del cuerpo que choca es $\left.\frac{d x}{d t}\right|_{t=0}=v_{0}$.

Bajo estas condiciones el objetivo[1] es determinar los parámetros $\kappa$ y $c$ que permiten que el valor máximo de $F$ sea un mínimo para un desplazamiento $x_{m}$ del parachoques lo más pequeño posible.

\subsection{Teorema PI de Buckingham}

Como todos los términos de una ecuación física son dimensionalmente homogéneos, es decir todos tiene la misma dimensión física, es posible dividir cada uno por las cantidades apropiadas para obtener una ecuación adimensional y para este caso particular con la ventaja que el número de parámetros se reduce a sólo uno, $\xi$. El proceso anterior genera un conjunto de parámetros adimensionales conocidos como los Pi's del problema[2][3] y se pueden obtener de forma metódica haciendo uso del teorema $\mathrm{Pi}$ de Buckingham de amplia aplicación en ingeniería y ciencias[4][5].

"El teorema Pi de Buckingham ${ }^{1}[3]$ establece que dadas $q_{1}, q_{2}, \ldots, q_{n}, n$ variables involucradas en un problema partícular, debe existir una relación funcional de la forma:

$$
f\left(q_{1}, q_{2}, \ldots, q_{n}\right)=0 .
$$

Entonces, siempre se pueden combinar estas $n$ variables para formar exactamente $n-j$ variables adimensionales independientes, donde $j$ es el rango de la matriz dimensional[3]. Cada parámetro adimensional es llamado un "número $\Pi$ ". Así la ecuación (2) puede ser escrita como la relación funcional:

$$
\phi\left(\Pi_{1}, \Pi_{2}, \ldots, \Pi_{n-j}\right)=0 .
$$

Se puede verificar rápidamente que estos parámetros adimensionales no son únicos. Sin embargo, $(n-j)$ de ellos son independientes y forman un conjunto completo[3].

Para implementarlo se proponen los siguientes pasos[2][3]: Realizar un lista de las variables dinámicas del problema. Este número se designa como $n$. Listar las dimensiones fundamentales necesarias para describir las $n$ variables dinámicas. A este número de variables fundamentales se denota con $j$. Del conjunto de $n$ variables dinámicas se escogen $j$ de ellas, que van a reemplazar las $j$ unidades fundamentales. Las $j$ nuevas variables dinámicas, se consideran como las nuevas fundamentales del problema a tratar. Quedan otras $n-j$ variables dinámicas, las cuales se podrían expresar en función de las $j$ nuevas variables fundamentales. El procedimiento a seguir consiste en hallar un parámetro adimensional asociado a cada una de las $n-j$ variables dinámicas, el cual se conoce como $\Pi_{i}$. Para ello

\footnotetext{
${ }^{1}$ Tomado textualmente de [4]
} 
se define estos $\Pi_{i}$ de la siguiente forma:

$$
\Pi_{i}=V_{D i} \prod_{k=1}^{j} V_{F k}^{a_{k}}, \quad \text { con: } i=1,2, \ldots n-j .
$$

Donde $V_{D}$ son las variables dinámicas y $V_{F}$ son las variables dinámicas que hacen las veces de variables fundamentales, y $\operatorname{los} a_{k}$ se escogen de tal forma que $\Pi_{i}$ sea adimensional. Finalmente se puede establecer una relación entre los $\Pi_{i}$, de la forma:

$$
\Pi_{1}=f\left(\Pi_{2}, \Pi_{3}, \ldots, \Pi_{n-j}\right) .^{\prime \prime}
$$

Para ver una aplicación relacionada con la física nuclear revisar[4][5].

$\mathrm{Al}$ aplicar el teorema $\mathrm{Pi}$ al problema del artículo:

\section{Variables dinámicas presentes en el problema}

1. La masa del objeto: $m$.

2. Constante de elásticidad del resorte: $\kappa$.

3. Coeficiente de disipación del amortiguador: $c$.

4. Desplazamiento de la masa: $x$.

5. Velocidad con que se aproxima la masa: $v$.

6. Fuerza sobre la masa: $F$.

7. Tiempo en que ocurre el evento: $t$.

Número de variables dinámicas: $n=7$.

\section{Magnitudes fundamentales relevantes en el problema}

1. Masa: $M$.

2. Longitud: $L$.

3. Tiempo: $T$.

Número de magnitudes fundamentales: $j=3$.

\section{Aplicación del Teorema $\Pi$}

De acuerdo al teorema $\Pi$ el número de parámetros adimensionales a buscar es: $m=n-j=7-3=4$. Se propone reemplazar $m, \kappa$ y $v$ en término de las magnitudes fundamentales y por lo tanto:

$$
\left\{\begin{array}{l}
\Pi_{1} \rightarrow x, \\
\Pi_{2} \rightarrow F \\
\Pi_{3} \rightarrow t \\
\Pi_{4} \rightarrow c .
\end{array}\right.
$$




\subsection{Cálculo de $\Pi_{1}$}

$\Pi_{1}$ es el parámetro adimensional relacionado con el desplazamiento, así:

$$
\Pi_{1}=x \cdot m^{a} \cdot \kappa^{b} \cdot v^{c},
$$

descomponiendo cada variable dinámica en sus correspondientes magnitudes fundamentales:

$$
\Pi_{1}=L \cdot M^{a} \cdot\left(\frac{M}{T^{2}}\right)^{b} \cdot\left(\frac{L}{T}\right)^{c}=L \cdot M^{a} \cdot \frac{M^{b}}{T^{2 b}} \cdot \frac{L^{c}}{T^{c}},
$$

agrupando:

$$
\Pi_{1}=L^{1+c} \cdot M^{a+b} \cdot T^{-2 b-c} .
$$

Garantizar adimensionalidad implica que:

$$
\begin{aligned}
1+c & =0, \\
a+b & =0, \\
-2 b-c & =0,
\end{aligned}
$$

que al solucionar lleva a:

$$
\begin{aligned}
& c=-1, \\
& a=-\frac{1}{2}, \\
& b=\frac{1}{2} .
\end{aligned}
$$

Reemplazando en la ecuación (6), se obtiene:

$$
\Pi_{1}=x \cdot m^{-1 / 2} \cdot \kappa^{1 / 2} \cdot v^{-1},
$$

así:

$$
\Pi_{1}=\frac{x}{v} \cdot \sqrt{\frac{\kappa}{m}} .
$$

Para valores típicos de $x$ y $v$ y considerando que $\omega=\sqrt{\kappa / m}$, finalmente:

$$
\Pi_{1}=\frac{x_{m} \omega}{v_{0}} .
$$

Calcular los otros $\Pi$ es repetir el procedimiento anterior, por lo tanto sólo se dirá que cada uno de estos Pi's corresponde a una magnitud relevante del problema respectivamente. De especial importancia para este problema son los tres primeros $\Pi$ 's, que se relacionan con variables que cambian en el tiempo y el tiempo mismo. Para reescribir la ecuación (1) con el fin que quede adimensional, se renombran 
los $\Pi$ 's de tal forma que la notación parezca más natural, así::

$$
\begin{array}{r}
\Pi_{1}=\frac{\omega}{v_{0}} x=z, \\
\Pi_{2}=\omega t=\tau, \\
\Pi_{3}=\frac{F x_{m}}{m v_{0}^{2}}=\bar{F}, \\
\Pi_{4}=\frac{c}{2 \sqrt{\kappa m}}=\xi,
\end{array}
$$

En esta nueva notación: $z$ juega el papel de $x$, el desplazamiento, pero sin dimensiones, $\tau$ el del tiempo $t$ y $\bar{F}$ el de la fuerza.

\section{Adimensionalización de la ecuación de movimiento}

Aplicando la regla de la cadena y teniendo en cuenta las relaciones funcionales dadas en la ecuación (8), se calcula:

$$
\frac{\partial z}{\partial \tau}=\frac{\partial z}{\partial x} \cdot \frac{\partial x}{\partial t} \cdot \frac{\partial t}{\partial \tau}=\stackrel{*}{z}
$$

al calcular las derivadas se llega a:

$$
\stackrel{*}{z}=\frac{\omega}{v_{0}} \dot{x} \frac{1}{\omega}=\frac{\dot{x}}{v_{0}},
$$

por lo tanto:

$$
\dot{x}=v_{0} \stackrel{*}{z}
$$

Para calcular la segunda derivada se debe tener en cuenta que $\stackrel{*}{z}$ depende de $\dot{x}$ (ecuación 9 ) y esta depende del tiempo $t$ y por las relaciones funcionales de la ecuación (8) el tiempo depende de $\tau$, así:

$$
\frac{\partial_{z}^{*}}{\partial \tau}=\frac{\partial_{z}^{*}}{\partial \dot{x}} \cdot \frac{\partial \dot{x}}{\partial t} \cdot \frac{\partial t}{\partial \tau}=\stackrel{* *}{z},
$$

derivando:

$$
\stackrel{* *}{z}=\frac{1}{v_{0}} \ddot{x} \frac{1}{\omega}=\frac{\ddot{x}}{\omega v_{0}}
$$

por lo tanto:

$$
\ddot{x}=\omega v_{0}{ }^{* *} \text {. }
$$

Despejando $x$ la primera ecuación de (8), obtiene:

$$
x=\frac{v_{0}}{\omega} z
$$

y reemplazando (11), (9) y (10) en la ecuación (1), lleva a:

$$
\omega v_{0} \stackrel{* *}{z}+\frac{c}{m} \underset{z}{z}+\omega^{2} \frac{v_{0}}{\omega} z=0
$$


que después de cancelar queda:

$$
\omega^{* *} z+\frac{c}{m} \stackrel{*}{z}+\omega z=0
$$

dividiendo entre $\omega$, se obtiene:

$$
\stackrel{* *}{z}+\frac{c}{m \omega} \stackrel{*}{z}+z=0
$$

y reemplazando $\omega$ por $\sqrt{\kappa / m}$, la ecuación anterior se transforma en:

$$
\stackrel{* *}{z}+\frac{c}{\sqrt{\kappa m}} \stackrel{*}{z}+z=0
$$

note que al multiplicar y dividir el segundo término aparece de forma natural $\Pi_{4}$, así:

$$
\stackrel{* *}{z}+2 \xi^{*}+z=0
$$

que es la equivalente adimensional a la ecuación dinámica del problema (ecuación 1), que además tiene la ventaja de tener un sólo parámetro[1]. Se puede verificar muy rápido que las condiciones iniciales son:

$$
\begin{aligned}
& z(0)=0 \\
& \stackrel{*}{z}(0)=1 .
\end{aligned}
$$

\section{Solución y análisis}

Para resolver la ecuación 12 se aplican los procedimientos tradicionales que se enseñan en los cursos de ecuaciones diferenciales. Así, la ecuación de índices de (12) es:

$$
r^{2}+2 \xi r+1=0
$$

con solución:

$$
r=-\xi \pm \sqrt{\xi^{2}-1}
$$

y se diferencian tres casos:

1. Si $\xi<1$, entonces: $r=-\xi \pm i \sqrt{1-\xi^{2}}$.

2. Si $\xi=1$, entonces: $r=-\xi$.

3. Si $\xi>1$, entonces: $r=-\xi \pm \sqrt{\xi^{2}-1}$.

\section{Caso 1: Exponenciales complejos}

Con $\xi<1$ la solución de la ecuación 12 es:

$$
z(\tau)=a_{1} e^{\left(-\xi+i \sqrt{1-\xi^{2}}\right) \tau}+a_{2} e^{\left(-\xi-i \sqrt{1-\xi^{2}}\right) \tau},
$$

factorizando,

$$
z(\tau)=e^{-\xi \tau}\left(a_{1} e^{i \sqrt{1-\xi^{2}} \tau}+a_{2} e^{-i \sqrt{1-\xi^{2}} \tau}\right)
$$


usando la identidad de Euler,

$$
\begin{aligned}
z(\tau) & =e^{-\xi \tau} a_{1}\left[\cos \left(\sqrt{1-\xi^{2}} \tau\right)+i \sin \left(\sqrt{1-\xi^{2}} \tau\right)\right] \\
& +e^{-\xi \tau} a_{2}\left[\cos \left(\sqrt{1-\xi^{2}} \tau\right)-i \sin \left(\sqrt{1-\xi^{2}} \tau\right)\right]
\end{aligned}
$$

agrupando,

$$
z(\tau)=e^{-\xi \tau}\left[\left(a_{1}+a_{2}\right) \cos \left(\sqrt{1-\xi^{2}} \tau\right)+i\left(a_{1}-a_{2}\right) \sin \left(\sqrt{1-\xi^{2}} \tau\right)\right],
$$

renombrando las constantes:

$$
z(\tau)=e^{-\xi \tau}\left[A_{1} \cos \left(\sqrt{1-\xi^{2}} \tau\right)+i A_{2} \sin \left(\sqrt{1-\xi^{2}} \tau\right)\right],
$$

para que se cumpla la condición $z(0)=0$ es claro que $A_{1}$ debe ser cero, por lo tanto:

$$
z(\tau)=i A_{2} e^{-\xi \tau} \sin \left(\sqrt{1-\xi^{2}} \tau\right)
$$

para encontrar el valor de $A_{2}$, se hace uso de la condición inicial $\stackrel{*}{z}(0)=1$, así:

$$
\begin{aligned}
\stackrel{*}{z}(0) & =\left.i A_{2}\left[-\xi e^{-\xi \tau} \sin \left(\sqrt{1-\xi^{2}} \tau\right)+e^{-\xi \tau} \cos \left(\sqrt{1-\xi^{2}} \tau\right) \sqrt{1-\xi^{2}}\right]\right|_{\tau=0} \\
& =i A_{2} \sqrt{1-\xi^{2}}=1,
\end{aligned}
$$

despejando,

$$
A_{2}=\frac{1}{i \sqrt{1-\xi^{2}}}
$$

reemplazando en la solución:

$$
z(\tau)=\frac{1}{\sqrt{1-\xi^{2}}} e^{-\xi \tau} \sin \left(\sqrt{1-\xi^{2}} \tau\right)
$$

\section{Caso 2}

Con $\xi=1$ la solución de la ecuación 12 es:

$$
z(\tau)=a_{1} e^{-\xi \tau}+a_{2} \tau e^{-\xi \tau}
$$

en la cual es inmediato que $a_{1}=0$ si se desea asegurar que $z(0)=0$, luego la solución se reduce a:

$$
z(\tau)=a_{2} \tau e^{-\xi \tau}
$$

derivando, evaluando en $\tau=0$ e igualando a 1 ,

$$
\stackrel{*}{z}(0)=\left.a_{2}\left(e^{-\xi \tau}-\tau \xi e^{-\xi \tau}\right)\right|_{\tau=0}=a_{2}=1,
$$

que concluye inmediatamente que:

$$
a_{2}=1 \text {, }
$$

reemplazando en la solución:

$$
z(\tau)=\tau e^{-\xi \tau}
$$




\section{caso 3}

Con $\xi>1$ la solución de la ecuación 12 es:

$$
z(\tau)=a_{1} e^{\left(-\xi+\sqrt{\xi^{2}-1}\right) \tau}+a_{2} e^{\left(-\xi-\sqrt{\xi^{2}-1}\right) \tau}
$$

factorizando:

$$
z(\tau)=e^{-\xi \tau}\left(a_{1} e^{\sqrt{\xi^{2}-1} \tau}+a_{2} e^{-\sqrt{\xi^{2}-1} \tau}\right)
$$

el lector puede comprobar que cambiando $a_{1}=\frac{A_{1}+A_{2}}{2}$ y $a_{2}=\frac{A_{1}-A_{2}}{2}$, la solución se puede reescribir como:

$$
z(\tau)=e^{-\xi \tau}\left[A_{1} \cosh \left(\sqrt{\xi^{2}-1} \tau\right)+A_{2} \sinh \left(\sqrt{\xi^{2}-1} \tau\right)\right]
$$

para asegurar que se cumpla que $z(0)=0$ se debe tomar $A_{1}=0$, luego:

$$
z(\tau)=A_{2} e^{-\xi \tau} \sinh \left(\sqrt{\xi^{2}-1} \tau\right)
$$

el valor de $A_{2}$ se obtiene al hacer $\stackrel{*}{z}(0)=1$, así:

$$
\begin{aligned}
\stackrel{*}{z}(0) & =\left.A_{2}\left[-\xi e^{-\xi \tau} \sinh \left(\sqrt{\xi^{2}-1} \tau\right)+e^{-\xi \tau} \cosh \left(\sqrt{\xi^{2}-1} \tau\right) \sqrt{\xi^{2}-1}\right]\right|_{\tau=0} \\
& =A_{2} \sqrt{\xi^{2}-1}=1
\end{aligned}
$$

luego:

$$
A_{2}=\frac{1}{\sqrt{\xi^{2}-1}}
$$

reemplazando en la solución,

$$
z(\tau)=\frac{1}{\sqrt{\xi^{2}-1}} e^{-\xi \tau} \sinh \left(\sqrt{\xi^{2}-1} \tau\right) .
$$

Al graficar las soluciones tomando para ello tres valores dentro de cada intervalo, se puede observar el siguiente comportamiento,

- El pico de la curva verde $\left(\xi_{v}=1\right)$ es fijo y tiene un valor entre 0.3 y 0.4 .

- El pico de la curva azul $\left(\xi_{a}=1.5>1\right)$ es inferior al de la curva verde y tiende al valor de la verde $\left(z_{a} \rightarrow z_{v}\right)$ cuando $\xi_{a} \rightarrow 1^{+}$.

- El pico de la curva roja $\left(\xi_{r}=0.5<1\right)$ es superior al de la curva verde y tiende al valor de la verde $\left(z_{r} \rightarrow z_{v}\right)$ cuando $\xi_{r} \rightarrow 1^{-}$.

De lo anterior se concluye directamente que el máximo de $z$ se consigue cuando $\xi<1$ y la solución del problema es la ecuación 15. Para encontrar este valor máximo se toma la derivada de la solución (ecuación 15) y se iguala a cero. En detalle:

$$
\frac{d z}{d \tau}=0
$$




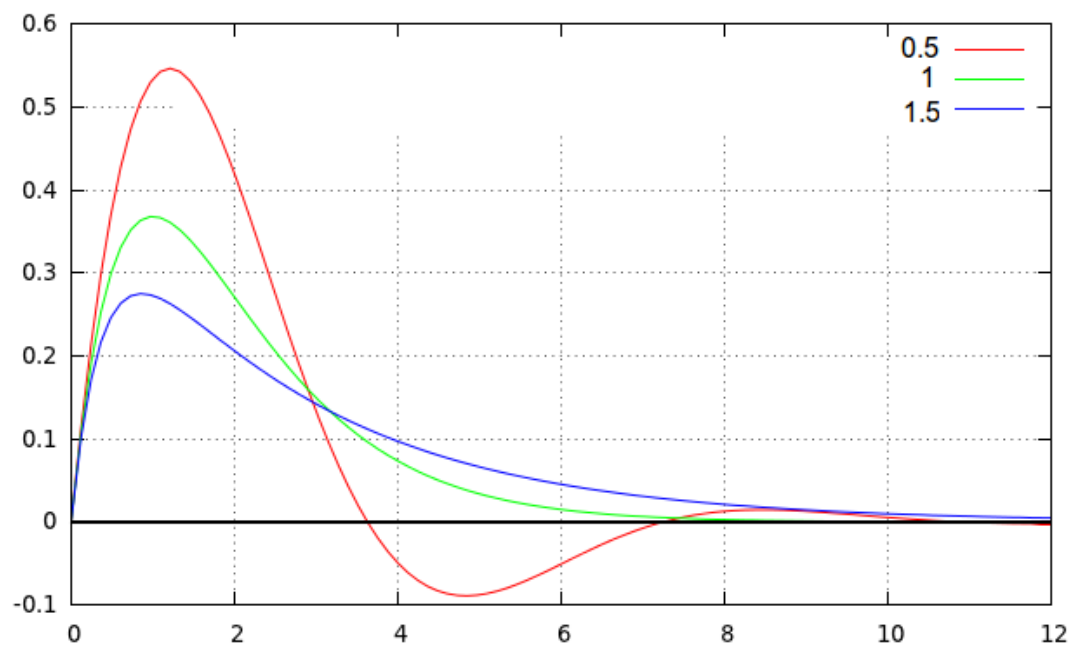

es decir:

$$
\frac{d}{d \tau}\left(\frac{1}{\sqrt{1-\xi^{2}}} e^{-\xi \tau} \sin \left(\sqrt{1-\xi^{2}} \tau\right)\right)=0
$$

derivando,

$$
\frac{1}{\sqrt{1-\xi^{2}}}\left[-\xi e^{-\xi \tau} \sin \left(\sqrt{1-\xi^{2}} \tau\right)+e^{-\xi \tau} \cos \left(\sqrt{1-\xi^{2}} \tau\right) \sqrt{1-\xi^{2}}\right]=0
$$

es cero lo que está dentro del paréntesis rectangular, así:

$$
-\xi e^{-\xi \tau} \sin \left(\sqrt{1-\xi^{2}} \tau\right)+\sqrt{1-\xi^{2}} e^{-\xi \tau} \cos \left(\sqrt{1-\xi^{2}} \tau\right)=0
$$

factorizando el exponente y resolviendo,

$$
-\xi \sin \left(\sqrt{1-\xi^{2}} \tau\right)+\sqrt{1-\xi^{2}} \cos \left(\sqrt{1-\xi^{2}} \tau\right)=0,
$$

lo que lleva a:

$$
\frac{\sin \left(\sqrt{1-\xi^{2}} \tau\right)}{\cos \left(\sqrt{1-\xi^{2}} \tau\right)}=\frac{\sqrt{1-\xi^{2}}}{\xi}
$$

y por lo tanto:

$$
\tan \left(\sqrt{1-\xi^{2}} \tau\right)=\frac{\sqrt{1-\xi^{2}}}{\xi}
$$

o mejor:

$$
\sqrt{1-\xi^{2}} \tau=\tan ^{-1}\left(\frac{\sqrt{1-\xi^{2}}}{\xi}\right)
$$




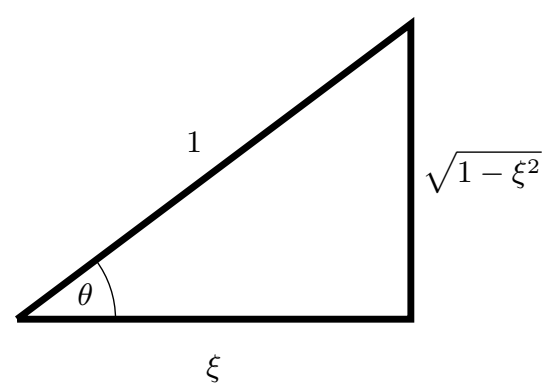

Figura 2. Triángulo que se construye a partir de la ecuación (18).

De acuerdo a la ecuación (18), los lados del triángulo que sugiere la tangente permiten deducir que la hipotenusa es 1, ver figura (2),

temporalmente se llamará $\theta$ a $\sqrt{1-\xi^{2}} \tau$. De acuerdo al dibujo se puede verificar que:

$$
\sin \theta=\frac{\sqrt{1-\xi^{2}}}{1}, \quad \text { por lo tanto } \theta=\sqrt{1-\xi^{2}} \tau=\sin ^{-1} \sqrt{1-\xi^{2}},
$$

así mismo,

$$
\cos \theta=\frac{\xi}{1}, \quad \text { por lo tanto } \theta=\sqrt{1-\xi^{2}} \tau=\cos ^{-1} \xi,
$$

y por lo tanto son válidas las siguientes relaciones:

$$
\sqrt{1-\xi^{2}} \tau=\tan ^{-1}\left(\frac{\sqrt{1-\xi^{2}}}{\xi}\right)=\sin ^{-1}\left(\sqrt{1-\xi^{2}}\right)=\cos ^{-1}(\xi),
$$

así el tiempo en que $z$ alcanza su valor máximo es:

$$
\tau=\frac{1}{\sqrt{1-\xi^{2}}} \tan ^{-1}\left(\frac{\sqrt{1-\xi^{2}}}{\xi}\right)=\tau_{f}
$$

que es particularmente útil escribirlo como:

$$
\tau_{f}=\frac{1}{\sqrt{1-\xi^{2}}} \sin ^{-1}\left(\sqrt{1-\xi^{2}}\right) .
$$

Reemplazando este tiempo en la ecuación (15),

$$
z\left(\tau_{f}\right)=\frac{1}{\sqrt{1-\xi^{2}}} e^{-\xi \tau_{f}} \sin \left(\sqrt{1-\xi^{2}} \frac{1}{\sqrt{1-\xi^{2}}} \sin ^{-1}\left(\sqrt{1-\xi^{2}}\right)\right)
$$

cancelando se llega finalmente a:

$$
z\left(\tau_{f}\right)=z_{m}=e^{-\xi \tau_{f}} .
$$


La fuerza normalizada se obtiene de la ecuación (12),

$$
\bar{F}=-\stackrel{* *}{z}=2 \xi^{* *} z+z
$$

o mejor:

$$
\bar{F}=-z_{m} \stackrel{* *}{z}=z_{m}\left(2 \xi^{* *}+z\right) .
$$

Antes de continuar se debe recordar que $\xi$ está restringido a valores entre $[0$ $\mathrm{y} 1]$.

\section{Optimizando $\xi$}

La ecuación (23) permite encontrar el mayor valor de la fuerza para cualquier $0<\xi<1$ y de todos estos valores interesa el mínimo. De acuerdo al problema este valor máximo de $\bar{F}$ está en el intervalo de tiempo (normalizado) $\tau=0$ y $\tau=\tau_{f}$, al inicio y final del recorrido de la masa. Entonces, en algún punto intermedio $\left(\tau=\tau_{m}\right)$ se cumple que:

$$
\frac{d \bar{F}}{d \tau}=0
$$

se debe recordar que las condiciones iniciales a la que se está sometido el sistema, son: $z(0)=0 \mathrm{y} \stackrel{*}{z}(0)=1$ y por consiguiente al final de recorrido: $z\left(\tau_{f}\right)=z_{m} \mathrm{y}$ $\stackrel{*}{z}\left(\tau_{f}\right)=0$.

Para calcular la fuerza en estos valores extremos, se hará uso de la ecuación (23), así:

- Al inicio del recorrido $(\tau=0)$,

$$
\bar{F}(0)=z_{m}\left(2 \xi_{z}^{*}(0)+z(0)\right)=z_{m}(2 \xi \cdot 1+0)=2 \xi z_{m} .
$$

- Al final del recorrido $\left(\tau=\tau_{f}\right)$,

$$
\bar{F}\left(\tau_{f}\right)=z_{m}\left(2 \xi^{*}\left(\tau_{f}\right)+z\left(\tau_{f}\right)\right)=z_{m}\left(2 \xi \cdot 0+z_{m}\right)=z_{m}^{2} .
$$

El mayor valor de la fuerza dentro del recorrido se encuentra calculando el tiempo $\tau_{m}\left(0<\tau_{m}<\tau_{f}\right)$ para el cual esta toma un valor máximo, y por lo tanto:

$$
\frac{d \bar{F}}{d \tau}=\bar{F}^{*}=0
$$

a partir de (23), lo anterior queda:

$$
\bar{F}^{*}=z_{m}\left(\stackrel{*}{z}+2 \xi^{* *} z\right)=0,
$$

derivando (15)

$$
\stackrel{*}{z}(\tau)=\frac{1}{\sqrt{1-\xi^{2}}}\left[-\xi e^{-\xi \tau} \sin \left(\sqrt{1-\xi^{2}} \tau\right)+\sqrt{1-\xi^{2}} e^{-\xi \tau} \cos \left(\sqrt{1-\xi^{2}} \tau\right)\right]
$$


distribuyendo,

$$
\stackrel{*}{z}(\tau)=-\xi \frac{1}{\sqrt{1-\xi^{2}}} e^{-\xi \tau} \sin \left(\sqrt{1-\xi^{2}} \tau\right)+e^{-\xi \tau} \cos \left(\sqrt{1-\xi^{2}} \tau\right) .
$$

Para facilitar el cálculo de la segunda derivada, la ecuación anterior se reescribe como:

$$
\stackrel{*}{z}(\tau)=-\xi z+e^{-\xi \tau} \cos \left(\sqrt{1-\xi^{2}} \tau\right)
$$

ahora se procede a calcular la segunda derivada, entonces:

$$
\stackrel{* *}{z}(\tau)=-\xi^{*}-\xi e^{-\xi \tau} \cos \left(\sqrt{1-\xi^{2}} \tau\right)-\sqrt{1-\xi^{2}} e^{-\xi \tau} \sin \left(\sqrt{1-\xi^{2}} \tau\right)
$$

se amplifica el segundo término, luego:

$$
\stackrel{* *}{z}(\tau)=-\xi^{*} z-\xi e^{-\xi \tau} \cos \left(\sqrt{1-\xi^{2}} \tau\right)-\left(1-\xi^{2}\right) \frac{1}{\sqrt{1-\xi^{2}}} e^{-\xi \tau} \sin \left(\sqrt{1-\xi^{2}} \tau\right),
$$

que gracias a (15) se puede reescribir como:

$$
\stackrel{* *}{z}(\tau)=-\xi^{*} z-\xi e^{-\xi \tau} \cos \left(\sqrt{1-\xi^{2}} \tau\right)-\left(1-\xi^{2}\right) z
$$

reemplazando (25) y (26) en (24),

$$
\begin{aligned}
& z_{m}\left[\left(-\xi z+e^{-\xi \tau} \cos \left(\sqrt{1-\xi^{2}} \tau\right)\right)\right. \\
& \left.+2 \xi\left(-\xi^{*}-\xi e^{-\xi \tau} \cos \left(\sqrt{1-\xi^{2}} \tau\right)-\left(1-\xi^{2}\right) z\right)\right]=0,
\end{aligned}
$$

distribuyendo,

$$
\begin{aligned}
& z_{m}\left[-\xi z+e^{-\xi \tau} \cos \left(\sqrt{1-\xi^{2}} \tau\right)\right. \\
& \left.-2 \xi^{2} z-2 \xi^{2} e^{-\xi \tau} \cos \left(\sqrt{1-\xi^{2}} \tau\right)-2 \xi z-2 \xi^{2} z\right]=0,
\end{aligned}
$$

agrupando los términos con coseno,

$$
z_{m}\left[-\xi z+\left(1-2 \xi^{2}\right) e^{-\xi \tau} \cos \left(\sqrt{1-\xi^{2}} \tau\right)-2 \xi^{2} z-2 \xi z-2 \xi^{2} z\right]=0,
$$

agrupando para factorizar por agrupación,

$$
z_{m}\left[-2 \xi z+\left(1-2 \xi^{2}\right) e^{-\xi \tau} \cos \left(\sqrt{1-\xi^{2}} \tau\right)-2 \xi^{2} z-\xi z\left(1-2 \xi^{2}\right)\right]=0,
$$

volviendo a factorizar,

$$
z_{m}\left[-2 \xi z+\left(1-2 \xi^{2}\right)\left[-\xi z+e^{-\xi \tau} \cos \left(\sqrt{1-\xi^{2}} \tau\right)\right]-2 \xi^{2} z\right]=0
$$

lo que está dentro del paréntesis cuadrado interno es $\stackrel{*}{z}$ (ecuación 25), por lo tanto,

$$
z_{m}\left[-2 \xi z+\left(1-2 \xi^{2}\right) \stackrel{*}{z}-2 \xi^{2} \underset{z}{z}\right]=0
$$


distribuyendo y sumando términos semejantes,

$$
z_{m}\left[-2 \xi z+\stackrel{*}{z}-2 \xi^{2} z-2 \xi^{2} z\right]=0
$$

ya que $z_{m}$ no es cero, entonces:

$$
-2 \xi z+\left(1-4 \xi^{2}\right) \stackrel{*}{z}=0
$$

reemplazando $z$ y $\stackrel{*}{z}$, ecuaciones (15) y (25) respectivamente,

$$
\begin{array}{r}
2 \xi\left(\frac{1}{\sqrt{1-\xi^{2}}} e^{-\xi \tau} \sin \left(\sqrt{1-\xi^{2}} \tau\right)\right)=\left(1-4 \xi^{2}\right) \\
\cdot\left(-\xi \frac{1}{\sqrt{1-\xi^{2}}} e^{-\xi \tau} \sin \left(\sqrt{1-\xi^{2}} \tau\right)+e^{-\xi \tau} \cos \left(\sqrt{1-\xi^{2}} \tau\right)\right)
\end{array}
$$

los exponentes se cancelan, distribuyendo, agrupando y factorizando se llega a:

$$
\left(\frac{\xi\left(3-4 \xi^{2}\right)}{\sqrt{1-\xi^{2}}}\right) \sin \left(\sqrt{1-\xi^{2}} \tau\right)=\left(1-4 \xi^{2}\right) \cos \left(\sqrt{1-\xi^{2}} \tau\right)
$$

transponiendo algunos factores,

$$
\frac{\sin \left(\sqrt{1-\xi^{2}} \tau\right)}{\cos \left(\sqrt{1-\xi^{2}} \tau\right)}=\frac{\sqrt{1-\xi^{2}}\left(1-4 \xi^{2}\right)}{\xi\left(3-4 \xi^{2}\right)},
$$

luego,

$$
\tan \left(\sqrt{1-\xi^{2}} \tau\right)=\frac{\sqrt{1-\xi^{2}}\left(1-4 \xi^{2}\right)}{\xi\left(3-4 \xi^{2}\right)},
$$

ecuación que permite despejar el tiempo $\left(\tau_{m}\right)$ en el cual la fuerza alcanza su valor máximo dentro del recorrido. Con la ecuación (27) se construye el triángulo que se muestra en la figura (3), llamando por comodidad $\theta$ al ángulo:

Note que en el triángulo la hipotenusa es 1 y esto permite las siguientes relaciones:

$$
\begin{aligned}
\sqrt{1-\xi^{2}} \tau & =\tan ^{-1}\left[\frac{\sqrt{1-\xi^{2}}\left(1-4 \xi^{2}\right)}{\xi\left(3-4 \xi^{2}\right)}\right] \\
& =\sin ^{-1}\left[\sqrt{1-\xi^{2}}\left(1-4 \xi^{2}\right)\right]=\cos ^{-1}\left[\xi\left(3-4 \xi^{2}\right)\right] .
\end{aligned}
$$

Así, el tiempo en el que la fuerza alcanza su valor máximo dentro del recorrido, es:

$$
\tau_{m}=\frac{1}{\sqrt{1-\xi^{2}}} \sin ^{-1}\left[\sqrt{1-\xi^{2}}\left(1-4 \xi^{2}\right)\right] .
$$




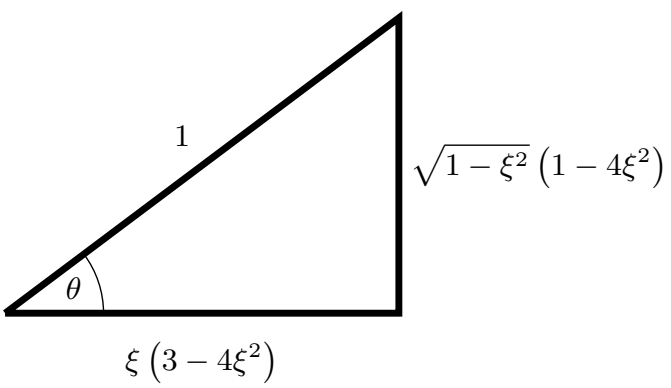

Figura 3. Triángulo que se construye a partir de la ecuación (27).

y este valor se reemplaza en la ecuación (23) para encontrar el valor máximo de la fuerza, así:

$$
\bar{F}_{m}=z_{m}\left(2 \xi^{*}\left(\tau_{m}\right)+z\left(\tau_{m}\right)\right),
$$

reemplazando $z$ y $\stackrel{*}{z}$, ecuaciones (15) y (25) respectivamente,

$$
\begin{aligned}
\bar{F}_{m} & =z_{m}\left[2 \xi\left(-\xi z\left(\tau_{m}\right)+e^{-\xi \tau_{m}} \cos \left(\sqrt{1-\xi^{2}} \tau_{m}\right)\right)\right. \\
& \left.+\frac{1}{\sqrt{1-\xi^{2}}} e^{-\xi \tau_{m}} \sin \left(\sqrt{1-\xi^{2}} \tau_{m}\right)\right]
\end{aligned}
$$

reemplazando apropiadamente las relaciones (28),

$$
\begin{aligned}
\bar{F}_{m} & =z_{m} e^{-\xi \tau_{m}}\left[\frac{\left(1-2 \xi^{2}\right)}{\sqrt{1-\xi^{2}}} \sin \left(\sqrt{1-\xi^{2}} \frac{1}{\sqrt{1-\xi^{2}}} \sin ^{-1}\left[\sqrt{1-\xi^{2}}\left(1-4 \xi^{2}\right)\right]\right)\right. \\
& \left.+2 \xi \cos \left(\sqrt{1-\xi^{2}} \frac{1}{\sqrt{1-\xi^{2}}} \cos ^{-1}\left[\xi\left(3-4 \xi^{2}\right)\right]\right)\right]
\end{aligned}
$$

aplicando las propiedades de cancelación del seno, lleva a:

$$
\bar{F}_{m}=z_{m} e^{-\xi \tau_{m}},
$$

y por la ecuación (22), la anterior queda:

$$
\bar{F}_{m}=e^{-\xi \tau_{f}} e^{-\xi \tau_{m}}
$$

$\mathrm{O}$

$$
\bar{F}_{m}=e^{-\xi\left(\tau_{f}+\tau_{m}\right)} .
$$

que corresponde al valor máximo que alcanza la fuerza dentro del recorrido en función de $\xi$. La tarea ahora consiste en encontrar para cuál valor de $\xi$ esta fuerza máxima toma su valor mínimo.

Note que la ecuación (29), restringe aún más los valores de $\xi$, por lo tanto:

$$
0 \leq \xi \leq \frac{1}{2}
$$

En los valores límites: 
- $\xi=0:$ A partir de (21) y reemplazando $\xi$ por cero, se obtiene:

$$
\tau_{f}=\frac{1}{\sqrt{1-0^{2}}} \tan ^{-1}\left(\frac{\sqrt{1-0^{2}}}{0}\right)=\frac{\pi}{2},
$$

y con (29),

$$
\tau_{f}=\frac{1}{\sqrt{1-0^{2}}} \tan ^{-1}\left(\frac{\sqrt{1-0^{2}}\left(1-4 \cdot 0^{2}\right)}{0 \cdot\left(3-4 \cdot 0^{2}\right)}\right)=\frac{\pi}{2},
$$

y de acuerdo a (30), la fuerza es:

$$
\bar{F}_{m}=e^{-0\left(\frac{\pi}{2}+\frac{\pi}{2}\right)}=1 .
$$

- $\xi=\frac{1}{2}$ : A partir de (21) y reemplazando $\xi$ por un medio, se obtiene:

$$
\tau_{f}=\frac{1}{\sqrt{1-\frac{1}{4}}} \tan ^{-1}\left(\frac{\sqrt{1-\frac{1}{4}}}{\frac{1}{2}}\right)=\frac{2}{\sqrt{3}} \tan ^{-1} \sqrt{3}=\frac{2 \pi}{3 \sqrt{3}},
$$

y $\operatorname{con}(29)$,

$$
\tau_{f}=\frac{1}{\sqrt{1-\frac{1}{4}}} \tan ^{-1}\left(\frac{\sqrt{1-\frac{1}{4}}\left(1-4 \cdot \frac{1}{4}\right)}{\frac{1}{2} \cdot\left(3-4 \cdot \frac{1}{4}\right)}\right)=0,
$$

y de acuerdo a (30), la fuerza es:

$$
\bar{F}_{m}=e^{-\frac{1}{2}\left(\frac{2 \pi}{3 \sqrt{3}}+0\right)} \approx 0.5463 .
$$

Al graficar la ecuación (30) en función de $\xi$, además de confirmar los valores extremos de arriba se puede verificar que $F_{m}$ alcanza el valor mínimo cuando $\xi$ está cerca de 0.4. Para encontrar el valor de $\xi$ que hace mínimo la fuerza máxima, se procede a calcular:

$$
\frac{d \bar{F}_{m}}{d \xi}=0
$$

así,

$$
\frac{d e^{-\xi\left(\tau_{f}+\tau_{m}\right)}}{d \xi}=0
$$

derivando:

$$
e^{-\xi\left(\tau_{f}+\tau_{m}\right)} \frac{d\left[-\xi\left(\tau_{f}+\tau_{m}\right)\right]}{d \xi}=0,
$$

como el exponente no es cero, entonces lo anterior se reduce a:

$$
-\frac{d\left[\xi\left(\tau_{f}+\tau_{m}\right)\right]}{d \xi}=0,
$$




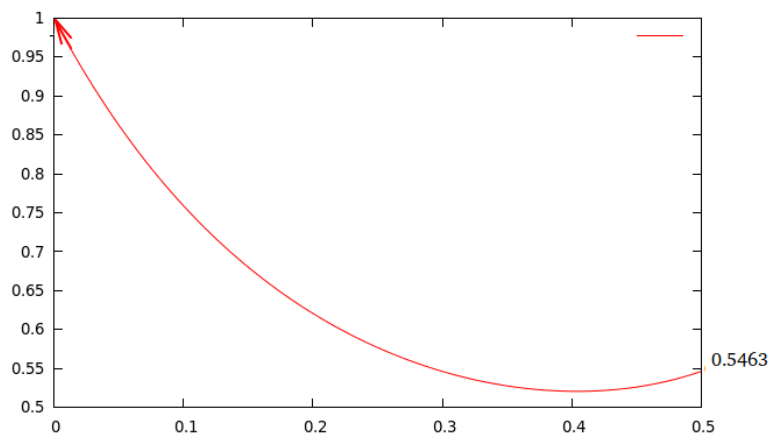

Figura 4. Gráfica de $F_{m}$ vs $\xi$. El valor mínimo de $F_{m}$ se encuentra cuando $\xi \approx 0.4$.

aplicando la regla del producto,

$$
\tau_{f}+\tau_{m}+\xi \frac{d}{d \xi}\left(\tau_{f}+\tau_{m}\right)=0
$$

propiedad lineal,

$$
\tau_{f}+\tau_{m}+\xi \frac{d \tau_{f}}{d \xi}+\xi \frac{d \tau_{m}}{d \xi}=0
$$

Se debe calcular cada derivada por aparte. Al reemplazar se llega a la ecuación:

$$
\tan ^{-1}\left(\frac{\sqrt{1-\xi^{2}}}{\xi}\right)+\tan ^{-1}\left[\frac{\sqrt{1-\xi^{2}}\left(1-4 \xi^{2}\right)}{\xi\left(3-4 \xi^{2}\right)}\right]-4 \xi \sqrt{1-\xi^{2}}=0,
$$

donde el valor de $\xi$ que hace mínima la fuerza normalizada se calcula por métodos numéricos con el algoritmo de Newton-Raphson. Para esto se toma:

$$
f(\xi)=\tan ^{-1}\left(\frac{\sqrt{1-\xi^{2}}}{\xi}\right)+\tan ^{-1}\left[\frac{\sqrt{1-\xi^{2}}\left(1-4 \xi^{2}\right)}{\xi\left(3-4 \xi^{2}\right)}\right]-4 \xi \sqrt{1-\xi^{2}} .
$$

se calcula la derivada para finalmente obtener la fórmula de iteración:

$$
\xi_{n+1}=\xi_{n}-\frac{\tan ^{-1}\left(\frac{\sqrt{1-\xi_{n}^{2}}}{\xi_{n}}\right)+\tan ^{-1}\left[\frac{\sqrt{1-\xi_{n}^{2}}\left(1-4 \xi_{n}^{2}\right)}{\xi_{n}\left(3-4 \xi_{n}^{2}\right)}\right]-4 \xi_{n} \sqrt{1-\xi_{n}^{2}}}{\frac{-8+8 \xi_{n}^{2}}{\sqrt{1-\xi_{n}^{2}}}},
$$

se toma como valor inicial $\xi=0.5$ ya que se sabe que $\xi$ debe estar cerca de 0.4 . Se implementa el código $\mathrm{C}++$ :

Método de Newton-Raphson 


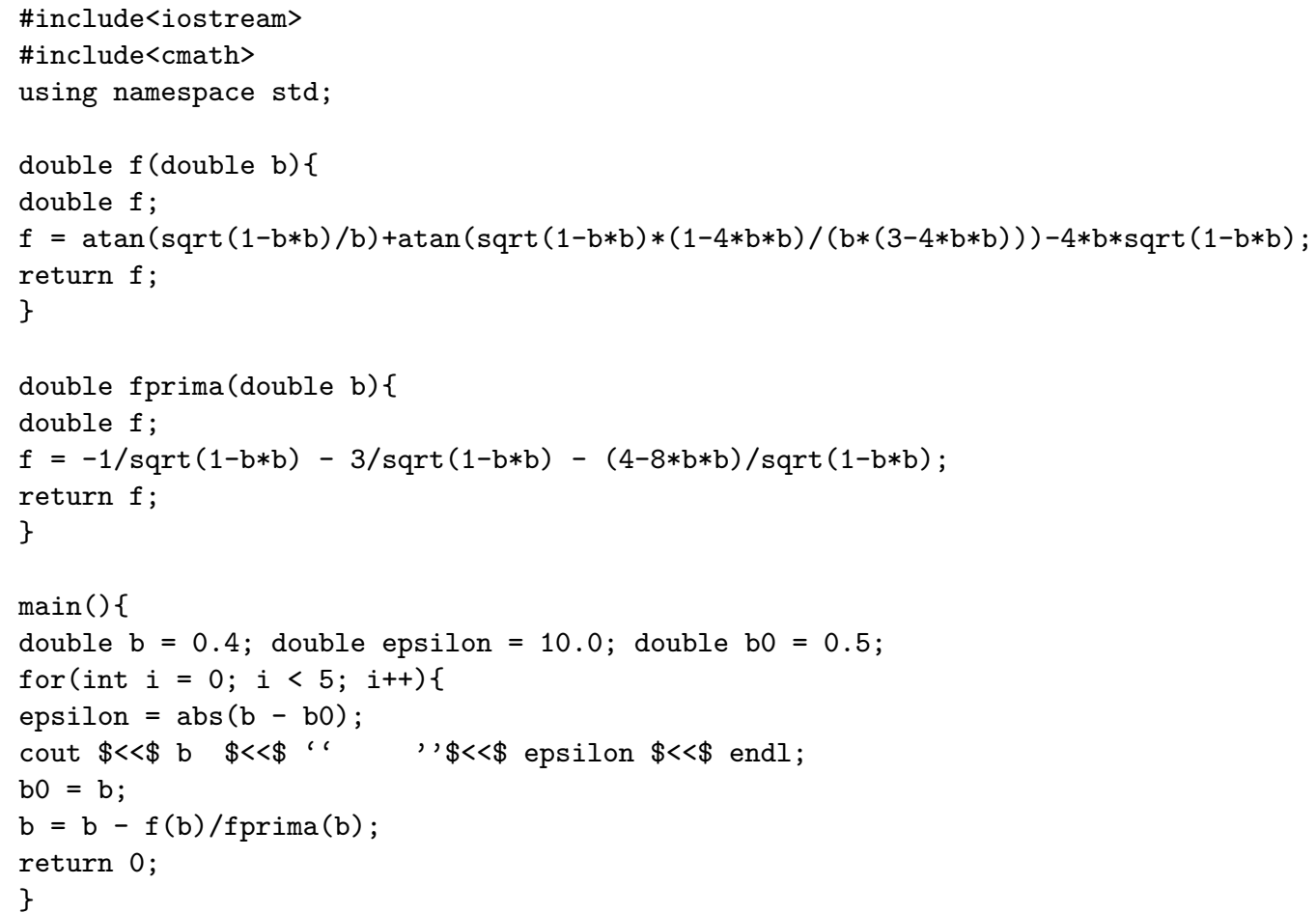

al ejecutar el programa se genera la siguiente tabla:

\begin{tabular}{ll}
$\mathrm{b}=\xi$ & epsilon \\
\hline 0.4 & 0.1 \\
0.403969 & 0.00396898 \\
0.403973 & $3.77263 \mathrm{e}-06$ \\
0.40397275 & $3.43542 \mathrm{e}-12$ \\
0.40397275 & 0 \\
\hline
\end{tabular}

\section{Cálculo de $\kappa$ y $c$ óptimos}

Para calcular el valor de la fuerza máxima primero se deben calcular los valores de $\tau_{f}$ y $\tau_{m}$, con las ecuaciones (20) y (29) respectivamente, así:

$$
\tau_{f}=\frac{1}{\sqrt{1-0.40397275^{2}}} \tan ^{-1}\left(\frac{\sqrt{1-0.40397275^{2}}}{0.40397275}\right) \approx 1.26254626,
$$

$\mathrm{y}$

$$
\begin{aligned}
\tau_{m} & =\frac{1}{\sqrt{1-0.40397275^{2}}} \tan ^{-1}\left(\frac{\sqrt{1-0.40397275^{2}}\left(1-4 \cdot 0.40397275^{2}\right)}{0.40397275\left(3-4 \cdot 0.40397275^{2}\right)}\right) \\
& \approx 0.35334476,
\end{aligned}
$$


y la fuerza máxima con la ecuación (30), así:

$$
\bar{F}_{m}=e^{-0.40397275(1.26254626+0.35334476)} \approx 0.52059862,
$$

y por lo tanto el valor de $z_{m}$ (ecuación (22)), es:

$$
z_{m}=e^{-0.40397275 \cdot 1.26254626} \approx 0.6004748 .
$$

En la siguiente tabla se resume el valor de los anteriores parámetros:

\begin{tabular}{cc} 
parámetro & valor \\
\hline$\xi$ & 0.40397275 \\
$\tau_{f}$ & 1.26254626 \\
$\tau_{m}$ & 0.35334476 \\
$\bar{F}_{m}$ & 0.52059862 \\
$z_{m}$ & 0.6004748. \\
\hline
\end{tabular}

Suponiendo que un obstáculo de $1000 \mathrm{~kg}$ se acerque a una rapidez de $8 \mathrm{~m} / \mathrm{s} \mathrm{y}$ que se estime un desplazamiento máximo del parachoques de $0.04 \mathrm{~m}$, es decir:

$$
\begin{aligned}
v_{0} & =8 \frac{\mathrm{m}}{\mathrm{s}}, \\
x_{m} & =0.04 \mathrm{~m}, \\
m & =1000 \mathrm{~kg},
\end{aligned}
$$

se calcula $\omega$, como:

$$
\omega=z_{m} \cdot \frac{v_{0}}{x_{m}}=0.6004748 \cdot \frac{8 \frac{\mathrm{m}}{\mathrm{s}}}{0.04 \mathrm{~m}} \approx 120.09496 \frac{\mathrm{rad}}{\mathrm{s}},
$$

y despejando $\kappa$ de la ecuación $\omega=\sqrt{\kappa / m}$, se obtiene el valor:

$$
\kappa=\omega^{2} \cdot m=\left(120.09496 \frac{\mathrm{rad}}{\mathrm{s}}\right)^{2} \cdot 1000 \mathrm{~kg} \approx 14422800 \frac{\mathrm{N}}{\mathrm{m}},
$$

finalmente de la definición de $\xi$ se despeja el valor de $c$, así:

$$
c=2 \xi \sqrt{\kappa \cdot m}=2 \cdot 0.40397275 \cdot \sqrt{14422800 \frac{\mathrm{N}}{\mathrm{m}} \cdot 1000 \mathrm{~kg}} \approx 97030.243 \frac{\mathrm{kg}}{\mathrm{s}} .
$$

Para resumir los últimos resultados se presenta la siguiente tabla:

\begin{tabular}{cr} 
parámetro & valor \\
\hline$\omega$ & $120.09496 \frac{\mathrm{rad}}{\mathrm{s}}$, \\
$\kappa$ & $14422800 \frac{\mathrm{N}}{\mathrm{m}}$, \\
$c$ & $97030.243 \frac{\mathrm{kg}}{\mathrm{s}}$.
\end{tabular}

Para la solución de 33 y la obtención de los valores óptimos, también se desarrolló en el instrumento virtual en Labview 8.5, del cual se muestra el panel frontal en la figura 5 y se aprecian los valores de las constantes $\kappa$ y $c$ para condiciones iniciales de desplazamiento, masa y velocidad. 


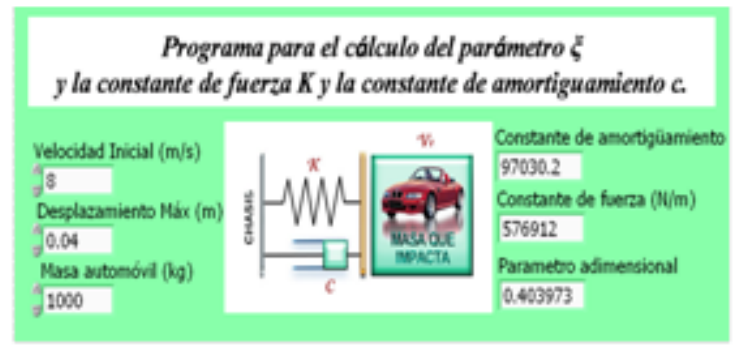

Figura 5. Panel frontal de VI desarrollado para el cálculo de $\xi, \kappa$ y $c$.

\section{Conclusión}

- El teorema Pi de Buckingham es una herramienta adecuada para formalizar la obtención de parámetros adimensionales que permiten la adimensionalización de ecuaciones diferenciales.

- Un problema complejo de ecuaciones con varios parámetros se simplifican de algún modo adimensionalizando la ecuación dinámica del sistema.

- En el proceso de adimensionalizar ecuaciones diferenciales se obtienen triángulos rectángulos de hipotenusa 1, figuras 2 y 3, que inciden directamente en la solución del problema.

\section{Referencias}

1. Peters D. A.: Optimum Spring-Damper Design for Mass Impact, SIAM Review, Vol. 39, No. 1 , pp. 118-122. (1997)

2. Cengel Y., Cimbala J.: Fluid Mechanics whith Studenr Resources. McGraw-Hill Science/engineering/Math; 2nd edition, (2009)

3. Kundu P. S., Cohen I. M.: Fluid Mechanics. Academic Press. (2002)

4. Bernal M., Martínez R., Cataño E.: Análisis Dimensional y Dispersión de Rutherford, RCF-2010. (2010)

5. Bernal M. A., Camacho F, J., Martinez R.: Dimensional analysis and Rutherford scattering, EUROPEAN JOURNAL OF PHYSICS, Eur. J. Phys. 34 L5?L8. (2013) 